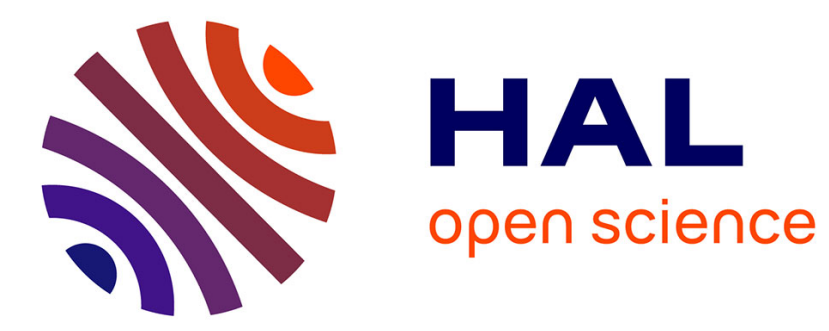

\title{
Intent-aware control in kinematically redundant systems: Towards collaborative wearable robots
}

Mahdi Khoramshahi, Guillaume Morel, Nathanael Jarrasse

\section{To cite this version:}

Mahdi Khoramshahi, Guillaume Morel, Nathanael Jarrasse. Intent-aware control in kinematically redundant systems: Towards collaborative wearable robots. 2021 IEEE International Conference on Robotics and Automation (ICRA), May 2021, Xi'an, China. hal-03181212

\section{HAL Id: hal-03181212 \\ https://hal.science/hal-03181212}

Submitted on 25 Mar 2021

HAL is a multi-disciplinary open access archive for the deposit and dissemination of scientific research documents, whether they are published or not. The documents may come from teaching and research institutions in France or abroad, or from public or private research centers.
L'archive ouverte pluridisciplinaire HAL, est destinée au dépôt et à la diffusion de documents scientifiques de niveau recherche, publiés ou non, émanant des établissements d'enseignement et de recherche français ou étrangers, des laboratoires publics ou privés. 


\title{
Intent-aware control in kinematically redundant systems: Towards collaborative wearable robots
}

\author{
Mahdi Khoramshahi, Guillaume Morel, and Nathanael Jarrassé
}

\begin{abstract}
Many human-robot collaboration scenarios can be seen as a redundant leader-follower setup where the human (i.e., the leader) can potentially perform the task without the assistance of the robot (i.e., the follower). Thus, the goal of the collaboration, beside stable execution of the task, is to reduce the human cost; e.g., ergonomic, or cognitive cost. Such system redundancies (where the same task be achieved in different manner) can also be exploited as a communication channel for the human to convey his/her intention to the robot; since it is essential for the overall performance (both execution and assistance) that the follower recognizes the intended task in an online fashion. Having an estimation for the intended task, the robot can assist the human by reducing the human cost over the task null-space; i.e., the null-space which arises from the overall system redundancies with respect to the intended task. With the prospective of supernumerary and prosthetic robots, in this work, we primarily focus on serial manipulation in which the proximal/distal part of the kinematic chain is controlled by the leader/follower respectively. By exploiting kinematic redundancies for intention-recognition and costminimization, our proposed control strategy (for the follower) ensures assistance under stable execution of the task. Our results (simulations and preliminary experimentation) show the efficacy of our method in providing a seamless robotic assistance (i.e., improving human posture) toward human intended tasks (i.e., reaching motions) for wearable robotics.
\end{abstract}

\section{INTRODUCTION}

Collaborative robotics has become the cynosure of research on pHRI (physical human-robot interaction) mainly due to its numerous applications: from industrial robotics (where mobile robots, manipulators, and humanoids aim to assist humans with cumbersome tasks [1-3]) to wearable robotics which aims to restore or augment human motor capacities (e.g., exoskeletons [4], prosthetic and supernumerary arms $[5,6])$. The ubiquitous properties of such applications are (1) the presence of heterogeneous agents, (2) decentralized control, and (3) redundancy: On one side of the interaction, we have an agent with higher cognitive capacities (often a human) who has the intention of performing a specific task, and on the other side, an agent with higher actuation capacity (often a robot) who aims to provide assistance. Decentralization occurs since the intention of the leader is latent to the follower and the communication channel is often limited to kinesthetics, myoelectric signals, etc. Furthermore, redundancy often arises from the fact that the human can potentially perform the task alone; robots

This work received funding from Swiss National Science Foundation (Grant number 191698) and ANR BYCEPS (ANR-18-CE19-0004).

${ }^{1}$ All authors are with Sorbonne Université, CNRS, INSERM, Institute for Intelligent Systems and Robotics (ISIR), Paris, France

* Corresponding author: khoramshahieisir.upmc.fr
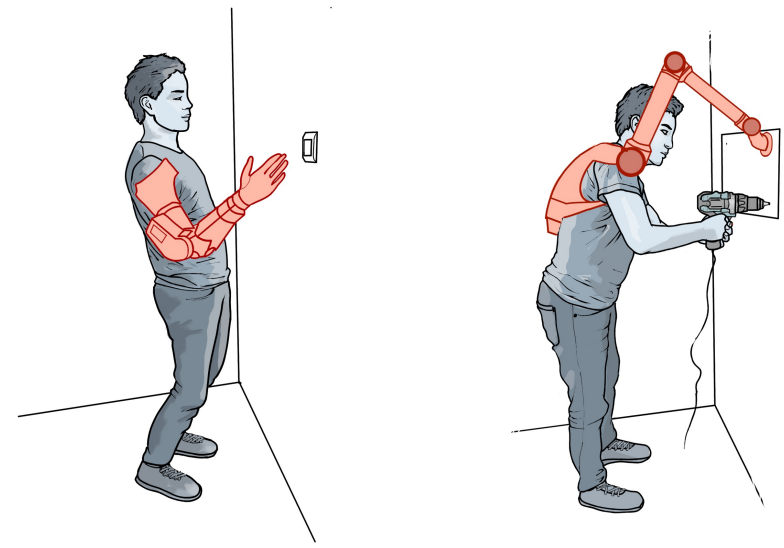

Fig. 1: Humans utilizing their kinematic redundancies to compensate for the lack of collaboration in wearable robots. In (a), the human leans backward to create an upward motion at the endeffector of the prosthetic arm, and in (b), leans forward to create forward motion at the end-effector of the supernumerary arm.

are employed to reduce human metabolic cost or improve human ergonomics, manipulability, etc. As an example, consider the case of a human-robot serial manipulation (illustrated in Fig. 1) where the human can perform his/her desired reaching motion even if the robot provides poor collaboration.

The major challenge for collaborative robots is to provide assistance toward an unknown task; i.e. the human-intended task. Methods relying on input devices to communicate the intentions (e.g., control panel, joystick, etc.) proved to be limited in their performance due to lack of realtime communication, necessity for extra hardware, or increase in human cognitive load [7-9]. Therefore, recent approaches aim at real-time integration of human intention (as discussed in [10]) based on motion [11, 12], forces $[13,14]$, impedance $[15,16]$, gesture $[17,18]$, etc. These approaches range from relying on delayed observation to prediction of human behavior which aims to increase robot's proactivity [11, 19-24]. Beside human-intention recognition, stability (and subsequently safety) is another challenge; i.e., to guarantee that the human-intended task is executed stably [24, 25]. Finally, the robotic input needs to be perceived as assistive by the human; e.g., consider an undesirable scenario where the human rejects robot's input as a disturbance to his/her intended task. In summary, any 
seamless collaborative control design requires: (1) robust human-intention recognition, (2) stable task execution, and (3) human cost minimization.

Numerous types of robotic assistance (i.e., cost function) are considered in the literature: reducing human effort in exerting a force [26], human tremor and oscillations [25], muscle fatigue [27], metabolic cost [28], etc. In this work, we primarily focus on assisting human to adopt an ergonomic posture; see [2, 29, 30] for postural optimization in human-robot co-manipulation tasks. Posture is also of particular interest to supernumerary and prosthetic robots since human users compromise their posture to compensate for their lack of direct control over non-natural joints [31]. Several conventional metrics exist for measuring ergonomic cost of a human posture such as REBA and RULA; see [32] as an application of such metrics. In this work, to focus on control aspect of our method, we assume that a leader preferred/ergonomic posture is given; e.g., having an upright torso. We show that stable convergence to both intended target position (of the reaching motion) and ergonomic posture can be achieved under our proposed control strategy.

In this work, we take inspiration from the literature of movement-based control of wearable device (particularly prosthetic arms) for impaired users. This line of research emerged to overcome the limitations of Myoelectric control [33]; mainly to decode human intention from surface EMG signal. Movement-based methods can be categorized into synergy-based and compensation cancellation control. The first category exploits the fact that there are kinematic synergies across joints in human reaching motion [34]. Therefore, the motion of robotic joints are coupled (via a synergy model) to human joints motion; e.g., prosthetic elbow coupled to human shoulder [35, 36]. This approach provides a reliable interface through which the human can indirectly control the robotic joint. Compensation cancellation control [37], however, leverages the fact that impaired users utilize their kinematic redundancies to create their desired motion at the end-effector; e.g., utilizing hip and shoulder to reach forward which compromises the ergonomics [38, 39]. Our previous works [40-42] show the effectiveness of movement-based approach (over Myoelectric control) in providing an ergonomic posture to the human user. However, these current approaches only operate in the joint-space and do not consider the humanintended task. In this work, we extend this line of research to a case where the robot recognizes the human-intended motions, which allows to provides assistance for both task execution and ergonomic posture. More specifically, we assume that measurements for the end-effector velocity and human joint positions are available for intention-recognition purposes. Furthermore, we analyze the performance of our proposed control strategy rigorously in terms of stability, convergence, and optimally.

\section{METHOD}

In this section, we first formulate our problem and later propose our control approach.

\section{A. Problem formulation}

Let us consider a generic n-DoF redundant robot with its joint configuration $q \in \mathbb{R}^{n}$ with its end-effector position $x \in$ $\mathbb{R}^{m}$ where $m<n$. We assume that the robot is controlled in a leader-follower fashion, where the task is only known to the leader. Furthermore, we assume that the first $l$ proximal joints are controlled by the leader $\left(q_{L}=\left[q_{1}, q_{2}, \ldots, q_{l}\right]\right)$ while the rest is controlled by the follower; i.e., $n-l$ distal joints $q_{F}=$ $\left[q_{l+1}, q_{l+2}, \ldots, q_{n}\right]$. To control the robot, the leader and the follower command their respective joint-velocities; i.e., $\dot{q}_{L}$ and $\dot{q}_{F}$ assuming perfect joint velocity tracking. Therefore, the velocity of the end-effector is obtained by using the task Jacobian:

$$
\dot{x}=\underbrace{\left[\begin{array}{ll}
J_{L} & J_{F}
\end{array}\right]}_{J}\left[\begin{array}{l}
\dot{q}_{L} \\
\dot{q}_{F}
\end{array}\right]
$$

The Jacobian matrix $\left(J(q) \in \mathbb{R}^{m \times n}\right)$ is segmented into respective parts for the leader $\left(J_{L} \in \mathbb{R}^{m \times l}\right)$ and the follower $\left(J_{F} \in \mathbb{R}^{m \times(n-l)}\right)$. We consider the following reaching task for the leader.

$$
v_{L}=-\gamma\left(x-x_{d}\right)
$$

where $x_{d}$ is the target position for the end-effector, $\gamma$ is a positive scalar, and $v_{L}$ is the leader desired task-velocity. Beside, we consider the following cost $\left(C \in \mathbb{R}^{+}\right)$:

$$
C=\frac{1}{2}(\dot{q}-\dot{\bar{q}})^{T} \underbrace{\left[\begin{array}{cc}
W_{L} & 0 \\
0 & W_{F}
\end{array}\right]}_{W}(\dot{q}-\dot{\bar{q}})
$$

where $\dot{\bar{q}}=-K(q-\hat{q})$ represents joint velocities toward desired posture $\hat{q} \in \mathbb{R}^{n}$ with $K \in \mathbb{R}^{n \times n}$ a positive definite matrix. $W_{L}$ and $W_{F}$ (positive definite matrices) account for the leader and follower weights respectively. The unified/centralized solution $\left(\dot{q}^{*}\right)$ to this problem (e.g., using method of Lagrange multipliers) is:

$$
\dot{q}^{*}=J^{\#} v_{L}+N \dot{\bar{q}}
$$

where the weighted pseudo-inverse of the Jacobian and its null-space projector can be segmented into the respective parts for the leader and the follower.

$$
\underbrace{\left[\begin{array}{c}
J_{L}^{\#} \\
J_{F}^{\#}
\end{array}\right]}_{J^{\#}}=W^{-1} J^{T}\left(J W^{-1} J^{T}\right)^{-1} \text {, and } \underbrace{\left[\begin{array}{c}
N_{L} \\
N_{F}
\end{array}\right]}_{N}=\left(I-J^{\#} J\right)
$$

However, in our leader-follower formulation, the follower is not aware of the task $\left(x_{d}\right.$ nor $\left.v_{L}\right)$, only the state of the system ( $q$ and $\dot{x}$ ) and the leader desired posture $(\hat{q})$ and the null-space gain $(K)$. In the next section, we propose our control strategy for solving this problem. 

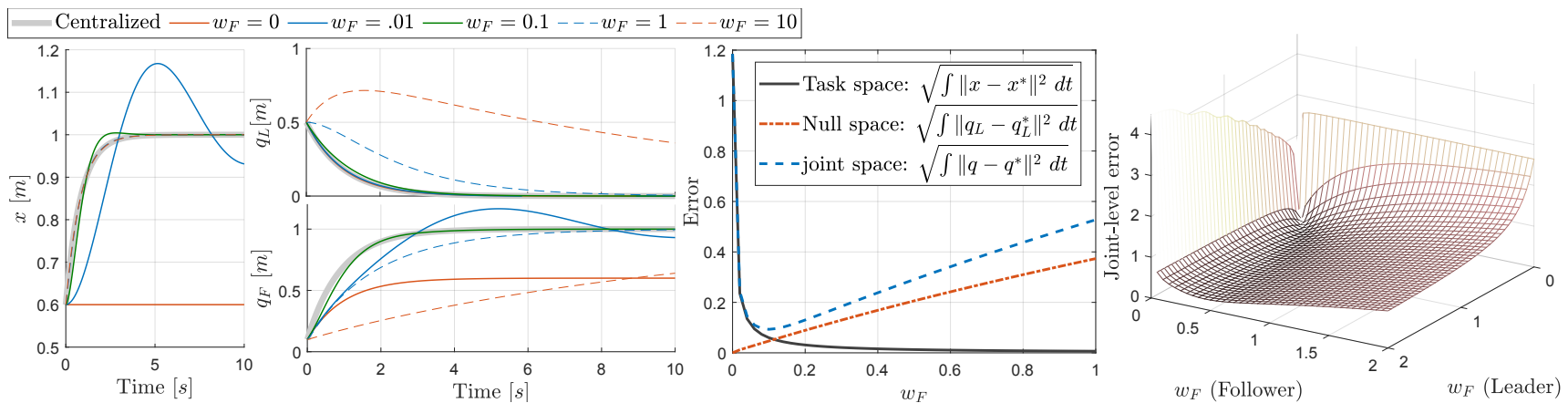

Fig. 2: The simulation results for the illustrative example of $n=2$ and $m=1$. The first plot shows the position of the end-effector under various values of $w_{F}$. the second plot show the joint position. The third plot shows how the performance is compared to a centralized solution for different values of $w_{F}$. The fourth plot shows the system performance when the leader and the follower have different assumption about $w_{F}$.

\section{B. Proposed control strategy}

We propose the following control strategy:

$$
\left\{\begin{array}{l}
\dot{q}_{L}=J_{L}^{\#} v_{L}+N_{L} \dot{\bar{q}} \\
\dot{q}_{F}=J_{F}^{\#} v_{F}+N_{F} \dot{\bar{q}} \\
\dot{v}_{F}=-\alpha\left(v_{F}-\dot{x}\right)
\end{array}\right.
$$

The first equation represents the leader's strategy which attempts to create its desired velocities $\left(v_{L}\right.$ and $\left.\dot{\bar{q}}\right)$. The second and third equations represent the follower's strategy which uses a lower-pass filtered version of $\dot{x}$ as an approximation for the leader intended velocity; i.e., $v_{F}$ is the follower inferred velocity, and $\alpha$ is positive scalar. In this strategy, the leader creates its desired velocity $\left(v_{L}\right)$ to reach the intended goal $\left(x_{d}\right)$, while the follower assists the leader via executing an inferred velocity $\left(v_{F}\right)$ based on previous observation of the end-effector motion $(\dot{x})$. Finally, let us note that the ODEs in Eq. 6 describe the desired dynamics where forward Euler method is used for numerical implementation.

In Appendix, we analyze the dynamics of the system under our proposed control strategy. In short, we show that when

1) the leader is not singular (i.e., capable of performing the task alone), and

2) the follower estimation rate is faster than the task dynamics (i.e., $\alpha>\gamma$ and $\alpha>K$ )

then

1) the task is achieved in a stable manner, and

2) the follower stably tracks (and converges to) the centralized solution (i.e., when the follower knows $v_{L}$ )

\section{ILLUSTRATIVE EXAMPLE}

Let us consider the most simple example where we have two prismatic joints and one-dimensional task $x=q_{L}+$ $q_{F}$. where $q_{L}$ and $q_{F}$ represent the leader and the follower joints respectively. Therefore, the Jacobian is $J=[1,1]$, which means $J_{L}=J_{F}=1$. For inverse kinematics (IK), we consider the following general parameterization:

$$
W=\left[\begin{array}{cc}
1 & 0 \\
0 & w_{F}
\end{array}\right] \rightarrow J^{\#}=\left[\begin{array}{l}
\bar{\lambda} \\
\lambda
\end{array}\right], N=\left[\begin{array}{cc}
\lambda & -\bar{\lambda} \\
-\lambda & \bar{\lambda}
\end{array}\right]
$$

with the follower IK weight $w_{F}>0, \lambda=1 /\left(1+w_{F}\right)$, and $\bar{\lambda}=1-\lambda$. For null-space velocity, we consider $\dot{\bar{q}}=$ $\left[-k q_{L}, 0\right]^{T}$, with $k>0$, in order to perform the task with $q_{L}=0$. In this case, the dynamics (derived from Eq. 6) are:

$$
\left\{\begin{array}{l}
\dot{q}_{L}=(1-\lambda) v_{L}-\lambda k q_{L} \\
\dot{q}_{F}=\lambda v_{F}+\lambda k q_{L} \\
\dot{v}_{F}=-\alpha\left(v_{F}-\dot{q}_{F}-\dot{q}_{L}\right)
\end{array}\right.
$$

At this point, it is interesting to consider two extreme cases:

$$
\left\{\begin{array}{l}
w_{F}=\infty \quad \rightarrow \quad \lambda=0 \quad \rightarrow \quad \dot{q}_{F}=0 \\
w_{F}=0 \rightarrow \lambda=1 \quad \rightarrow \quad \dot{q}_{F}=-\dot{q}_{L}
\end{array}\right.
$$

It is intuitive to see that in the case of $w_{F}=\infty$, zero velocities are expected from the follower as it has a high cost. On the other hand, when $w_{F}=0$, the leader is not performing any portion of the task since it is expected that the follower performs the task completely. Therefore, the follower recognizes $v_{L}=0$ as the intended motion. The follower moves only in order to cancel the leader's motion on the end-effector $(\dot{x}=0)$ as the leader still moves in order to reach $q_{L}=0$. For other cases (where $0<\lambda<1$ ), we can write (by taking the time-derivative of $\dot{q}_{F}$ in Eq. 8):

$$
\ddot{q}_{F}=-\alpha \bar{\lambda}\left(\dot{q}_{F}-\dot{q}_{F}^{*}-\Delta \dot{q}_{F}\right)
$$

where $\dot{q}_{F}^{*}=\lambda v_{L}+\lambda k q_{L}$ represent the centralized solution for the follower, and $\Delta \dot{q}_{F}=k \alpha^{-1}\left(\lambda v_{L}-\lambda^{2} \bar{\lambda}^{-1} k q_{L}\right)$ accounts for the error induced by filtering (i.e., intention estimation). This error is mitigated by higher values for $\alpha$. Moreover, since $-\alpha \bar{\lambda}<0$, the follower velocity converges to $\dot{q}_{F}^{*}$ (when $\Delta \dot{q}_{F}$ can be neglected). These dynamics also show that small values of $\lambda$ lead to faster convergence to the centralized solution. However, in centralized solutions with low $\lambda$ (i.e., high $w_{F}$ ), the follower contributes less (i.e., $\dot{q}_{F}^{*} \propto \lambda$ ). Therefore, in the decentralized scenario, we face a trade-off between intention-recognition and assistance; where the leader is required to perform the task partially in order to communicate the intended goal to the follower and subsequently receive assistance. 


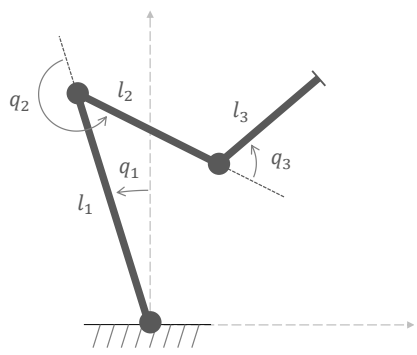

(a)

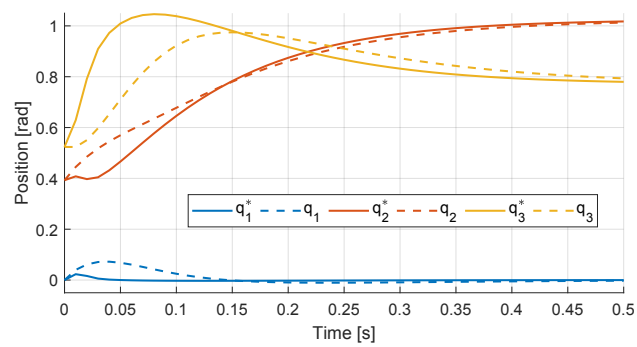

(b)

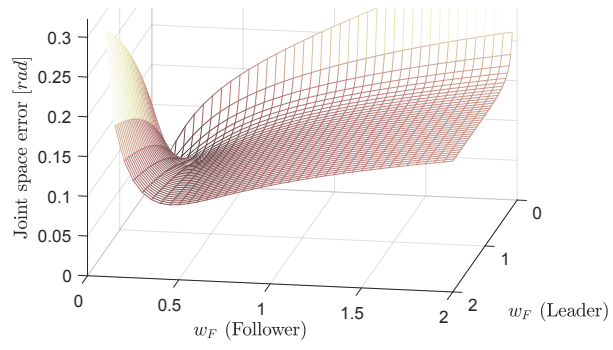

(c)

Fig. 3: The simulation results for the 3-DoF planar manipulator. (a) The model. (b) The joint values. (c) The joint-level error (compared to the centralized solution) when the agents have different assumptions about each other's contribution (i.e., $w_{F}$ ).

Fig 2 shows the results of a simulation for this 1-D case. We simulate the system behavior for different values of $w_{F}$ and compare against the centralized solution. For reaching task, we consider $v_{L}=-1.2(x-1)$ and $k=1$ for the null-space. We initialized the system at $q_{L}=0.5, q_{F}=0.1$, and $v_{F}=0$, and all their derivatives at zero. The centralized solution is generated with $w_{F}=0$ and $v_{F}=v_{L}$. The results show the aforementioned trade-off. Higher values of $w_{F}$ lead to similar results to those of the centralized solution at the end-effector; see the first subplot. However, lower values of $w_{F}$ perform similarly in the null-space; see $q_{L}$ in the second subplot. Finally, the third subplot $\left(q_{F}\right)$ shows that with $w_{F}=0.1, q_{F}$ behaves similar to $q_{F}^{*}$. The fourth subplot shows this trade-off in a quantitative manner where $w_{F}$ has opposite effect on task and null-space error.

It is crucial to investigate the case where the leader and the follower have different assumptions about each other's contribution. Note that in our formulation, we assumed that both agents utilize the same gain matrix $W$ in their inverse kinematics. Last subplot in Fig. 2 shows the performance of our control approach (in terms of joint-level error) where the leader and the follower have different assumption for $w_{F}$. Intuitively, the leader assuming small values or the follower assuming big values (i.e., relying heavily on the other partner) are counter-productive. When the follower assumes $w_{F}<1$ (i.e., to contribute more than the leader), the performance is not only satisfactory, but also less sensitive to the leader's assumption.

\section{THREE-LINKS PLANAR ROBOT}

In this simulation, we consider a 3-DoF planar model illustrated in Fig. 3a where the first two joints are controlled by the leader and the last joint by the follower. This resembles a simplified model for human reaching in the sagittal plane utilizing hip, shoulder, and a prosthetic elbow. In this model, we have $l_{1}=0.6, l_{2}=0.3$, and $l_{3}=0.25 \mathrm{~m}$. The task is to reach $x_{d}=[.5, .5]^{T}$ with $v_{L}=-0.1\left(x-x_{d}\right)$. The preferred posture is $\hat{q}=[0,0,0]^{T}$ with $K=\operatorname{diag}[2,0,0]$; i.e., only keeping the first link upright. The robot is initialized at $q=[0 ;-7 \pi / 8 ; \pi / 6]$ and simulated with $d t=0.01$. The centralized solution is generated using $W=\operatorname{diag}([1, .1, .05])$, whereas in the leader-follower scenario $W=\operatorname{diag}([1, .1, .5])$. We choose $w_{F}=0.5$ (lower expected contribution from the follower) to investigate the performance of the robust case which we expect following the insight from the previous results presented in Fig. 2.

The results presented in Fig. $3 \mathrm{~b}$ compares the centralized and collaborative scenarios in terms of joint angles. As expected by the weight matrix $W$ in the centralized scenario, the reaching motion is mostly achieved by moving the third and the second joint. However, in the collaborative scenario, the leader moves its joints $\left(q_{1}\right.$ and $\left.q_{2}\right)$ more to convey the intended motion. Interestingly, the leader only uses $q_{1}$ on the onset of the motion and goes back to its preferred value. Compared to the centralized case, the follower motion has less amplitude (due to higher $w_{F}$ ) and is delayed (due to filtering). It can be seen that after $0.2 \mathrm{~s}$, the joint values converge to the centralized solution. Furthermore, Fig. 3c shows the joint-space error (compared to the centralized solution) when the leader and the follower have different assumption about $w_{F}$. The graph is similar to the one obtained from the 1-DoF example; i.e. the results are less sensitive to the leader's assumption when the follower assumes $w_{F}=0.4$. However, a lower error can be achieved if both agree upon $w_{F}=0.1$.

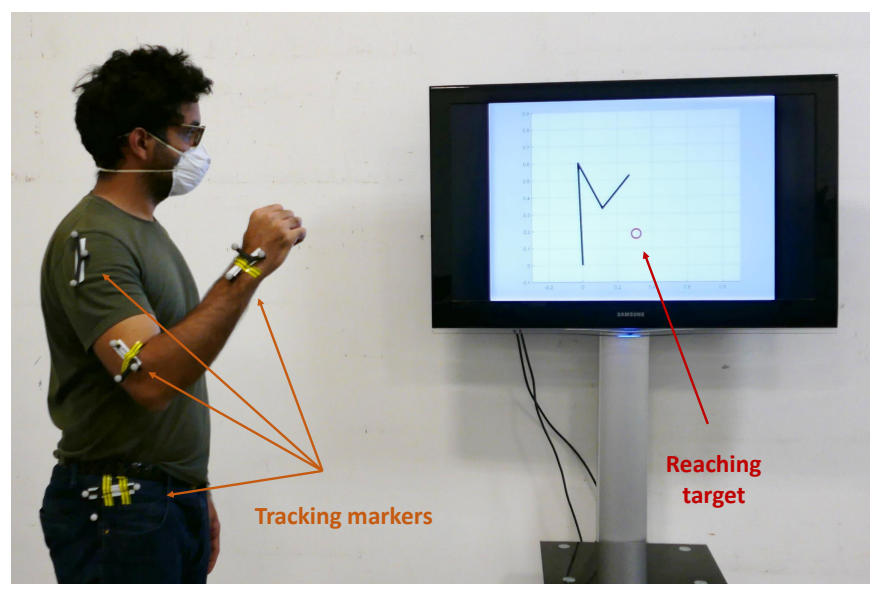

Fig. 4: The experimental setup where a human subject controls a kinematic chain on the screen with his/her respective joints in order to reach a designated target. We use this setup to compare different control strategies for the third joint. 

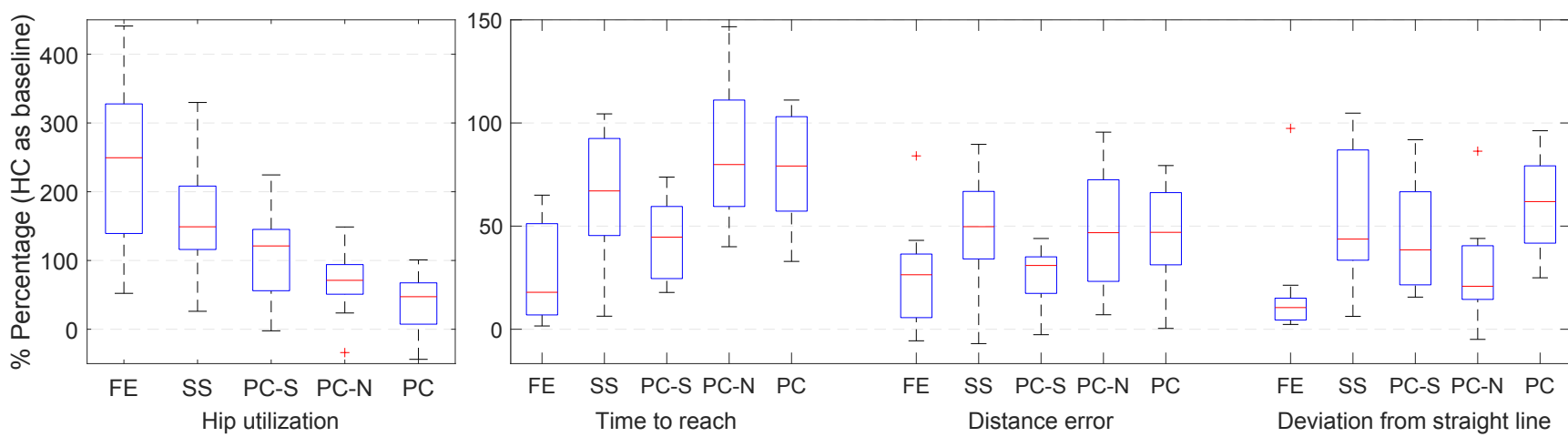

Fig. 5: The experimental result where humans controls the first 2 joints and different strategies used for the third joint. The results are presented in percentages in comparison to the $\mathrm{HC}$ condition where the human controls all the three joints. Each distribution is presented in terms of min, 25th percentile, median, 75th percentile, and max. Outliers are indicated by ' + ' symbol.

\section{EXPERIMENTAL RESULTS}

In this section, we validate our proposed control method facing a leader with higher complexity. To do so, we asked 10 participants to control the 3-DoF manipulator (of the previous section) with their respective kinematic chain in the sagittal plane; hip, shoulder, and elbow to control $q_{1}, q_{2}$, and $q_{3}$ respectively. We use the Optitrack system to track four marker-clusters attached to the subject upper body (hip, shoulder, elbow and wrist) from which we obtains the values for $q$. The participants were asked to reach for a series of target positions in the frontal reachable workspace. These targets position, as well as the $3-\mathrm{DoF}$ robot, was displayed to the participants on a monitor screen as illustrated in Fig. 4.

To better investigate our approach, we considered the following conditions. In $H C$, the participant controls all the tree joints; i.e., a centralized control. For $F E$, the third joint (elbow) is fixed at $q_{3}=\frac{\pi}{6}$, and the participant controls $q_{1}$ and $q_{2}$. In $S S, q_{3}$ is controlled using a simple shoulder-elbow synergy; i.e., $\dot{q}_{3}=\dot{q}_{2}$ where $q_{1}$ and $q_{2}$ is controlled by the subject. In $P C$, the proposed control method is used to control the third joint. For $P C-S$, only the synergy part of the propose method is used; i.e., $\dot{\bar{q}}=0$ in Eq. 6. Finally in $P S-N$, only the null-space part of the proposed method is used; i.e., $v_{F}=0$ in Eq. 6. The sequence of the target position (consists of $G=28$ goals) were kept fixed across the conditions. We designed this sequence to accommodate motions in different direction; since the previous reached-goal will be the initial position to reach another. Each target was presented for a period of $6 s$ making the trial $T=168 \mathrm{~s}$ long. Prior to each trial, the participants had a short training session to familiarize with the control strategy. For our controller, we used $\alpha=40, W=\operatorname{diag}([1,1, .15]), K=\operatorname{diag}([2, .2,0])$, and $\hat{q}=[0,-2.7,0] \mathrm{rad}$. Finally, the control loop frequency, in average, was around $50 \mathrm{~Hz}$.

We consider the four following metric (listed in the table below) to evaluate the performance of participants. Hip utilization is computed as the average absolute value of the first joint $\left(q_{1}\right)$. Distance error is obtained as the average of distance between the end-effector and the target $e=x-x_{d}$ where $\|$.$\| denotes the vector norm. Time to reach is the$ average time spent to reach a target position with tolerance $\delta=0.02 m$ : Finally, Deviation from straight line is the average of the velocity components that are orthogonal to the error vector $e$. The participants' performances across conditions are summarized in Fig. 5. In this figure, the performance of each participant in each conditions is compared (in percentages) to their individual performances in the $\mathrm{HC}$ condition.

\begin{tabular}{lll} 
Metric & Equation & Average in HC \\
\hline \hline Hip utilization & $\frac{1}{T} \int_{0}^{T}\left\|q_{1}(t)\right\| d t$ & $0.075 \mathrm{rad}$ \\
\hline Distance error & $\frac{1}{T} \int_{0}^{T}\|e\| d t$ & $0.07 \mathrm{~m}$ \\
\hline $\begin{array}{l}\text { Time to reach } \\
\frac{1}{G} \int_{\|e\|>\delta} d t\end{array}$ & $2.5 \mathrm{~s}$ \\
\hline $\begin{array}{l}\text { Deviation from } \\
\text { straight line }\end{array}$ & $\frac{1}{T} \int_{0}^{T}\left\|\dot{x}-\frac{\dot{x}^{T} e}{\|e\|} e\right\| d t$ & $0.05 \mathrm{~m} / \mathrm{s}$
\end{tabular}

The obtained results show that our approach is effective in reducing the hip utilization in human participants; $47 \%$ compared to $250 \%$ when the elbow is locked, and $150 \%$ when the simple synergy method is used. However, it can be seen that the equality of reaching motion (in terms of the other three metrics) is worse compared to $H C$ and $F E$ when the human (even though the third joints is locked) controls the system in a centralized manner. Such lower performance is expected since in the decentralized control the follower has uncertainties (about the intended task and over control parameters) and suffers from delayed observations. Nevertheless, comparison between $S S$ and $P C$ reveals that more complex dynamics (which reduces the hip utilization by $102 \%$ ) does not exacerbate the quality of reaching drastically; $10 \%$ slower reaching with $17 \%$ higher deviation from straight line. Moreover, the results shows that due to the faster dynamics of the synergy part of our method, the target is reached faster but with higher deviation from the straight line. On the other hand, slower dynamics of the null-space 
part lead to less deviation but slower reaching is achieved. These observations are useful in tuning parameters such as $w_{F}$ and $K$ which adjust the reactivity of each respective part. Finally, it is important to note the variability among participants (as the distributions have high variance) which hints at 1) personalized parameter tuning, and 2) possibility for improvement after training.

\section{DISCUSSION AND CONCLUSION}

In this work, we present an intent-aware control method for collaborative robots in kinematically redundant setups with applications towards prosthetic and supernumerary arms. Our proposed method is inspired by the movement-based control approach in which it was observed that impaired users utilize their kinematic redundancies (and subsequently, compromise the ergonomics of their posture) to perform their intended task. Our method exploits the fact that the intended task can be recognized through the observations of the end-effector velocity created by the leader. We formulate our method based on the segmentation of the Jacobian matrix which allowed us to investigate the stability and convergence behavior rigorously. The simulation results are in line with the theoretical aspects of the method and shed light on how certain parameters affect the final performance; e.g., $w_{F}$ as the follower IK gain which sets a trade-off between optimality and robustness.

Our experimental results show that our proposed method is effective in recognizing the intended task, and thus, reducing the ergonomic cost for human-leaders. We compare our method against a simple linear synergy model which often used in the literature. Most often, the synergy model is extracted from healthy subjects $([43,44])$ to be used for individuals with amputations ([36, 45]). However, such methods neglect the kinematic changes in individuals with amputations; e.g., higher hip and scapula utilization to compensate for the lack of control over the distal joints. Moreover, we put our method under further scrutiny by investigating the performance of the separate parts of the controller; namely the synergy and the null-space part in Eq. 6. The obtained results hint at how the respective gains of each part $\left(w_{F}\right.$ and $\left.K\right)$ can be tuned effectively in order to provide satisfactory dynamics for the human-user; slow dynamics increase the reaching time and fast dynamics cause fluctuations and overshoots.

The comparison between simulation and experimental results show that better performance is achieved when the leader and the follower have complementary dynamics; i.e., the leader and the follower solving the IK problem by using the respective parts of a unified Jacobian. However, in the experimental scenario, the unmodeled/unexpected dynamics seen by each agents deteriorates the tracking performance. In our future work, we will try to improve our approach by accommodating human-like dynamics; e.g., through online learning and personalization. Moreover, in future, we will implement our method on a prosthetic arm where we need to tackle technical challenges such as embedded and noisy measurements, imperfect physical coupling and robot dynamics.

\section{APPENDIX}

1) Proof of stability: The dynamics of this system can be studied by plugging Eq. 6 into Eq. 1, which results in

$$
\dot{x}=-\gamma H_{L}\left(x-x_{d}\right)+H_{F} v_{F}
$$

where $H_{L}=J_{L} J_{L}^{\#}$ and $H_{F}=J_{F} J_{F}^{\#}$ with property that $H_{L}+H_{F}=J J^{\#}=I ; I$ being the identity matrix. The dynamics of the error can also be investigated with respect to the target position; i.e., $e=x-x_{d}$ and its time-derivative:

$$
\dot{e}=-\gamma H_{L} e+H_{F} v_{F}
$$

The second time-derivative yields the dynamics as

$$
\ddot{e}+(\gamma+\alpha) H_{L} \dot{e}+\alpha \gamma H_{L} e=\eta_{x}
$$

where the $\eta_{x}=\dot{H}_{L}\left(\lambda e-v_{F}\right)$. Since $\gamma$ and $\alpha$ are positive scalars, the homogeneous system is stable if all eigenvalues of $H_{L}$ are positive. To show this, we expand $H_{L}$ as follows.

$$
H_{L}=J_{L} W_{L}^{-1} J_{L}^{T}\left(J_{L} W_{L}^{-1} J_{L}^{T}+J_{F} W_{F}^{-1} J_{F}^{T}\right)^{-1}
$$

Here, we assume that $J_{L} W_{L}^{-1} J_{L}^{T}$ is invertible; i.e., the leader is not singular. Therefore, $H_{L}$ can be simplified to:

$$
H_{L}=(I+A)^{-1}
$$

where $A=\left(J_{F} W_{F}^{-1} J_{F}^{T}\right)\left(J_{L} W_{L}^{-1} J_{L}^{T}\right)^{-1}$ has non-negative eigenvalues since it is the multiplication of a semi-positive and a positive definite matrix. Therefore, if $\lambda \geq 0$ is eigenvalue for $A, \frac{1}{1+\lambda}>0$ is an eigenvalue for $H_{L}$.

The previous analysis shows that the task can be achieve in a stable manner. However, we also need to show that internal dynamics are stable; e.g., the undesirable behavior when the leader compensates for the follower's unstable dynamics. Thus, we study the follower's dynamics which are

$$
\ddot{q}_{F}=-\alpha\left(I-J_{F}^{\#} J_{F}\right)\left(\dot{q}_{F}-\dot{q}_{F}^{*}\right)+\eta_{q}
$$

where $\dot{q}_{F}^{*}=J_{F}^{\#} v_{L}+N_{F} \dot{\bar{q}}$ represent the centralized solution of the follower; where $v_{F}$ is replaced by $v_{L}$. Moreover, $\eta_{q}=N_{F} \ddot{\bar{q}}+\dot{N}_{F} \dot{\bar{q}}+\dot{J}_{F}^{\#} v_{F}$ represents the disturbances due to higher order dynamics. The matrix $J_{F}^{\#} J_{F}$ has the same non-zero eigenvalues of $H_{F}$. Since $H_{L}+H_{F}=I$, it follows that $\left(I-J_{F}^{\#} J_{F}\right)$ has eigenvalues of form $\frac{1}{1+\lambda}>0$ as well. This guarantees a stable convergence of $\dot{q}_{F}$ to its centralized solution when $\eta_{q}$ can be neglected.

Finally, in our analysis, we assume a perfect velocitycontroller and we ignore the second-order dynamics of the system; e.g., inertia, centrifugal forces and gravity. However, such neglected discrepancies can be accommodated into $\eta_{x}$ and $\eta_{q}$ as disturbances that can be tolerated by the velocity controller. Our analysis (at the velocity level) and proposed method (on motion planning for the follower) still hold even if one imagines other types of controllers such as torquelevel control (e.g., inverse dynamics control [46]) as long as the control objective is to track $\dot{q}_{F}$ proposed by Eq. 6 . 


\section{REFERENCES}

[1] B. Busch, G. Cotugno, M. Khoramshahi, G. Skaltsas, D. Turchi, L. Urbano, M. Wächter, Y. Zhou, T. Asfour, G. Deacon et al., "Evaluation of an industrial robotic assistant in an ecological environment," in 2019 28th IEEE International Conference on Robot and Human Interactive Communication (RO-MAN). IEEE, 2019, pp. 1-8.

[2] K. Wansoo, M. Lorenzini, B. Pietro, W. Yuqiang, and A. Ajoudani, "Towards ergonomic control of collaborative effort in multi-human mobile-robot teams," in IEEE/RSJ International Conference on Intelligent Robots and Systems, 2019.

[3] D. J. Agravante, A. Cherubini, A. Sherikov, P.-B. Wieber, and A. Kheddar, "Human-humanoid collaborative carrying," IEEE Transactions on Robotics, vol. 35, no. 4, pp. 833-846, 2019.

[4] R. Goehlich, M. Rutsch, and I. Krohne, "Magic triangle-human, exoskeleton, and collaborative robot scenario," Technische Unterstützungssysteme, die die Menschen wirklich wollen, p. 435, 2018.

[5] S.-w. Leigh, H. Agrawal, and P. Maes, "Robotic symbionts: Interweaving human and machine actions," IEEE Pervasive Computing, vol. 17, no. 2, pp. 34-43, 2018.

[6] F. Parietti and H. Asada, "Supernumerary robotic limbs for human body support," IEEE Transactions on Robotics, vol. 32, no. 2, pp. 301-311, 2016.

[7] A. Steinfeld, T. Fong, D. Kaber, M. Lewis, J. Scholtz, A. Schultz, and M. Goodrich, "Common metrics for human-robot interaction," in Proceedings of the 1st ACM SIGCHI/SIGART conference on Humanrobot interaction, 2006, pp. 33-40.

[8] V. Villani, F. Pini, F. Leali, and C. Secchi, "Survey on human-robot collaboration in industrial settings: Safety, intuitive interfaces and applications," Mechatronics, vol. 55, pp. 248-266, 2018.

[9] D. P. Losey, C. G. McDonald, E. Battaglia, and M. K. O’Malley, "A review of intent detection, arbitration, and communication aspects of shared control for physical human-robot interaction," Applied Mechanics Reviews, vol. 70, no. 1, 2018.

[10] A. Ajoudani, A. M. Zanchettin, S. Ivaldi, A. Albu-Schäffer, K. Kosuge, and O. Khatib, "Progress and prospects of the human-robot collaboration," Autonomous Robots, vol. 42, no. 5, pp. 957-975, 2018.

[11] D. Aarno and D. Kragic, "Motion intention recognition in robot assisted applications," Robotics and Autonomous Systems, vol. 56, no. 8, pp. 692-705, 2008.

[12] J. Huang, W. Huo, W. Xu, S. Mohammed, and Y. Amirat, "Control of upper-limb power-assist exoskeleton using a human-robot interface based on motion intention recognition," IEEE transactions on automation science and engineering, vol. 12, no. 4, pp. 1257-1270, 2015.

[13] A. Kucukyilmaz, T. M. Sezgin, and C. Basdogan, "Intention recognition for dynamic role exchange in haptic collaboration," IEEE transactions on haptics, vol. 6, no. 1, pp. 58-68, 2012.

[14] A. Olivares-Alarcos, S. Foix, and G. Alenya, "On inferring intentions in shared tasks for industrial collaborative robots," Electronics, vol. 8, no. 11, p. 1306, 2019.

[15] Y. Li, G. Ganesh, N. Jarrassé, S. Haddadin, A. Albu-Schaeffer, and E. Burdet, "Force, impedance, and trajectory learning for contact tooling and haptic identification," IEEE Transactions on Robotics, vol. 34, no. 5, pp. 1170-1182, 2018.

[16] Z. Liu and J. Hao, "Intention recognition in physical human-robot interaction based on radial basis function neural network," Journal of Robotics, vol. 2019, 2019.

[17] F. Chen, H. Lv, Z. Pang, J. Zhang, Y. Hou, Y. Gu, H. Yang, and G. Yang, "Wristcam: A wearable sensor for hand trajectory gesture recognition and intelligent human-robot interaction," IEEE Sensors Journal, vol. 19, no. 19, pp. 8441-8451, 2018.

[18] C. Zhu and W. Sheng, "Wearable sensor-based hand gesture and daily activity recognition for robot-assisted living," IEEE Transactions on Systems, Man, and Cybernetics-Part A: Systems and Humans, vol. 41, no. 3, pp. 569-573, 2011.

[19] J. Mainprice and D. Berenson, "Human-robot collaborative manipulation planning using early prediction of human motion," in 2013 IEEE/RSJ International Conference on Intelligent Robots and Systems. IEEE, 2013, pp. 299-306.

[20] G. Maeda, M. Ewerton, R. Lioutikov, H. B. Amor, J. Peters, and G. Neumann, "Learning interaction for collaborative tasks with probabilistic movement primitives," in 2014 IEEE-RAS International Conference on Humanoid Robots. IEEE, 2014, pp. 527-534.

[21] J. Mainprice, R. Hayne, and D. Berenson, "Goal set inverse optimal control and iterative replanning for predicting human reaching motions in shared workspaces," IEEE Transactions on Robotics, vol. 32, no. 4, pp. 897-908, 2016.

[22] H. Liu and L. Wang, "Human motion prediction for human-robot collaboration," Journal of Manufacturing Systems, vol. 44, pp. 287294, 2017.

[23] M. Khoramshahi, A. Laurens, T. Triquet, and A. Billard, "From human physical interaction to online motion adaptation using parameterized dynamical systems," in 2018 IEEE/RSJ International Conference on Intelligent Robots and Systems (IROS). IEEE, 2018, pp. 1361-1366.

[24] M. Khoramshahi and A. Billard, "A dynamical system approach to task-adaptation in physical human-robot interaction," Autonomous Robots, vol. 43, no. 4, pp. 927-946, 2019.

[25] V. Duchaine and C. Gosselin, "Safe, stable and intuitive control for physical human-robot interaction," in 2009 IEEE International Conference on Robotics and Automation. IEEE, 2009, pp. 33833388.

[26] C. T. Landi, F. Ferraguti, L. Sabattini, C. Secchi, and C. Fantuzzi, "Admittance control parameter adaptation for physical human-robot interaction," in 2017 IEEE international conference on robotics and automation (ICRA). IEEE, 2017, pp. 2911-2916.

[27] L. Peternel, C. Fang, N. Tsagarakis, and A. Ajoudani, "A selective muscle fatigue management approach to ergonomic human-robot co-manipulation," Robotics and Computer-Integrated Manufacturing, vol. 58, pp. 69-79, 2019.

[28] R. Nasiri, A. Ahmadi, and M. N. Ahmadabadi, "Reducing the energy cost of human running using an unpowered exoskeleton," IEEE Transactions on Neural Systems and Rehabilitation Engineering, vol. 26, no. 10, pp. 2026-2032, 2018.

[29] C. Thomas, L. Stankiewicz, A. Grötsch, S. Wischniewski, J. Deuse, and B. Kuhlenkötter, "Intuitive work assistance by reciprocal humanrobot interaction in the subject area of direct human-robot collaboration," Procedia Cirp, vol. 44, pp. 275-280, 2016.

[30] B. Busch, G. Maeda, Y. Mollard, M. Demangeat, and M. Lopes, "Postural optimization for an ergonomic human-robot interaction," in 2017 IEEE/RSJ International Conference on Intelligent Robots and Systems (IROS). IEEE, 2017, pp. 2778-2785.

[31] A. J. Metzger, A. W. Dromerick, R. J. Holley, and P. S. Lum, "Characterization of compensatory trunk movements during prosthetic upper limb reaching tasks," Archives of physical medicine and rehabilitation, vol. 93, no. 11, pp. 2029-2034, 2012.

[32] A. Dwyer, J. Huckleby, M. Kabbani, A. Delano, M. De Sutter, and D. Crawford, "Ergonomic assessment of robotic general surgeons: a pilot study," Journal of Robotic Surgery, pp. 1-6, 2019.

[33] P. Geethanjali, "Myoelectric control of prosthetic hands: state-of-theart review," Medical Devices (Auckland, NZ), vol. 9, p. 247, 2016.

[34] T. Bockemühl, N. F. Troje, and V. Dürr, "Inter-joint coupling and joint angle synergies of human catching movements," Human Movement Science, vol. 29, no. 1, pp. 73-93, 2010.

[35] M. Popovic and D. Popovic, "Cloning biological synergies improves control of elbow neuroprostheses," IEEE Engineering in Medicine and Biology Magazine, vol. 20, no. 1, pp. 74-81, 2001.

[36] R. Garcia-Rosas, Y. Tan, D. Oetomo, C. Manzie, and P. Choong, "Personalized online adaptation of kinematic synergies for humanprosthesis interfaces," IEEE transactions on cybernetics, 2019.

[37] M. Legrand, M. Merad, E. de Montalivet, A. Roby-Brami, and N. Jarrassé, "Movement-based control for upper-limb prosthetics: Is the regression technique the key to a robust and accurate control?" Frontiers in neurorobotics, vol. 12, p. 41, 2018.

[38] F. Montagnani, M. Controzzi, and C. Cipriani, "Exploiting arm posture synergies in activities of daily living to control the wrist rotation in upper limb prostheses: a feasibility study," in 2015 37th Annual International Conference of the IEEE Engineering in Medicine and Biology Society (EMBC). IEEE, 2015, pp. 2462-2465.

[39] A. Hussaini, A. Zinck, and P. Kyberd, "Categorization of compensatory motions in transradial myoelectric prosthesis users," Prosthetics and orthotics international, vol. 41, no. 3, pp. 286-293, 2017.

[40] M. Merad, É. de Montalivet, A. Roby-Brami, and N. Jarrassé, "Intuitive prosthetic control using upper limb inter-joint coordinations and imu-based shoulder angles measurement: a pilot study," in 2016 IEEE/RSJ International Conference on Intelligent Robots and Systems (IROS). IEEE, 2016, pp. 5677-5682.

[41] M. Merad, É. De Montalivet, A. Touillet, N. Martinet, A. RobyBrami, and N. Jarrassé, "Can we achieve intuitive prosthetic elbow control based on healthy upper limb motor strategies?" Frontiers in neurorobotics, vol. 12, p. 1, 2018. 
[42] M. Merad, E. de Montalivet, M. Legrand, E. Mastinu, M. OrtizCatalan, A. Touillet, N. Martinet, J. Paysant, A. Roby-Brami, and N. Jarrasse, "Assessment of an automatic prosthetic elbow control strategy using residual limb motion for transhumeral amputated individuals with socket or osseointegrated prostheses," IEEE Transactions on Medical Robotics and Bionics, vol. 2, no. 1, pp. 38-49, 2020.

[43] R. Vinjamuri, V. Patel, M. Powell, Z.-H. Mao, and N. Crone, "Candidates for synergies: linear discriminants versus principal components," Computational intelligence and neuroscience, vol. 2014, 2014.

[44] N. Lambert-Shirzad and H. M. Van der Loos, "On identifying kinematic and muscle synergies: a comparison of matrix factorization methods using experimental data from the healthy population," Journal of neurophysiology, vol. 117, no. 1, pp. 290-302, 2017.

[45] N. A. Alshammary, D. A. Bennett, and M. Goldfarb, "Efficacy of coordinating shoulder and elbow motion in a myoelectric transhumeral prosthesis in reaching tasks," in 2016 IEEE International Conference on Robotics and Automation (ICRA). IEEE, 2016, pp. 3723-3728.

[46] W. K. Chung, L.-C. Fu, and T. Kröger, "Motion control," in Springer Handbook of Robotics. Springer, 2016 